Research Article

\title{
An FBG Strain Sensor-Based NPW Method for Natural Gas Pipeline Leakage Detection
}

\author{
Qingmin Hou $(\mathbb{D}$ \\ School of Energy and Building Engineering, Harbin University of Commerce, Harbin 150028, China \\ Correspondence should be addressed to Qingmin Hou; sspawn@163.com
}

Received 8 January 2021; Revised 18 February 2021; Accepted 10 March 2021; Published 15 March 2021

Academic Editor: Haoran Zhang

Copyright (c) 2021 Qingmin Hou. This is an open access article distributed under the Creative Commons Attribution License, which permits unrestricted use, distribution, and reproduction in any medium, provided the original work is properly cited.

\begin{abstract}
Natural gas pipeline leaks can lead to serious and dangerous accidents that can cause great losses of life and property. Therefore, detecting natural gas pipeline leaks has always been an important subject. The negative pressure wave (NPW) method is currently the most widely used leakage detection method. Generally, this method uses pressure sensors to detect NPW signals to assess the leak and determine the location of the leakage point. However, the installation of a pressure sensor requires penetrating the pipeline structure, so the sensor intervals are often distant, leading to large signal attenuations and the ineffective detection of small leaks. An NPW method based on fiber Bragg grating (FBG) strain sensors is proposed in this paper which detects NPWs by monitoring the annular strain of the pipeline. Moreover, due to the advantages of nondestructive installation FBG strain sensors can be arranged closer along the distance of the pipeline, the attenuation of the NPW is small and the detection of leaks is improved. This method is tested through experiments and compared with a pressure sensor-based method; the experimental results verify that the proposed method is more effective in detecting natural gas pipeline leaks.
\end{abstract}

\section{Introduction}

Natural gas pipelines are the preferred method of natural gas transmission. However, as gas is a hazardous material, a leaking or broken gas pipeline can cause great harm to the surrounding environment and personnel. When a gas pipeline leaks, especially a high-pressure gas pipeline compressed gas will expand rapidly and release a large amount of energy causing explosions and fires that can lead to considerable economic losses and casualties.

The service life of pipelines is similar to the lifespan of human beings which can be roughly divided into three stages: infancy ( $1 \sim 5$ years old) adulthood ( $6 \sim 20$ years old), and old age (over 20 years old). Correspondingly, the service process of pipelines can also be divided into these three stages. When the pipeline is in its infancy, the probability of accidents, especially leakage, is very low. When the pipeline is in middle age, the accident rates are relatively stable and can be kept at a low level. When the pipeline is old, the accident rates are higher and tend to increase over time.
There are many methods in use for gas pipeline leak detection but the principles and technical means of each method are different [1-7]. The pressure sensor-based negative pressure wave (NPW) method is widely used in this field because it can avoid the establishment of a complex mathematical model for the pipelines and is easy to operate [8]. This method is effective for the detection of sudden leaks but not for small leaks that occur slowly. This method is easily disturbed by external factors and it exhibits a high failure rate.

Detection technology based on distributed fiber sensors is widely used at present to detect pipeline leaks [9-20]. In this method, a fiber is arranged along the pipeline to determine whether the pipeline is leaking and to locate the leaking position by detecting the vibration or temperature change caused by the leak. However, due to the complex external environment of buried pipelines, they may cross urban areas, rivers, and so on. Many external factors may also lead to vibration or temperature changes such as rolling vehicles, random optical fiber vibrations, and climate change. Therefore, this method is prone to 
interference has a high false alarm rate and can only determine an abnormal condition in a pipeline. At present, there are few applications of quasi-distributed optical fiber Bragg grating (FBG) used in the detection of natural gas pipeline leaks [21-25]. Lopez et al. developed an FBG sensor based on an expandable polymer that can expand when it encounters oil and thus produces strain [26]. However, because the main component of natural gas is methane which has stable chemical properties that hinder the expansion of a polymer, this sensor is not suitable for the detection of natural gas pipeline leaks.

In this paper, an idea is proposed. An NPW generated by a natural gas pipeline leak will cause a pressure variation in the pipeline; this pressure variation will create an annular strain in the pipeline and the pipeline leak can be detected by monitoring the annular strain of the pipeline through an FBG strain sensor. Based on the abovementioned principles, an FBG strain sensor-based NPW method for natural gas pipeline leak detection is proposed and this method is tested and compared with an experiment.

\section{Pressure Sensor-Based NPW Method}

Based on the theory of fluid mechanics, it can be seen that the pressure in a natural gas pipeline tends to be stable overall although there are some small fluctuations before the leak. When a pipeline leak occurs due to the pressure difference between the inside and outside of the pipeline, the fluid at the leak point will escape rapidly and the fluid density at the leak point will decrease, resulting in an instantaneous pressure drop. This instantaneous pressure drop propagates as a wave up and down the leak point. If the pressure inside the pipe under normal conditions is taken as the reference standard, the pressure wave generated by the leak is an NPW. With the generation and propagation of the disturbance, the NPW will reduce the pressure along the pipeline.

2.1. Principle of Pressure Sensor-Based NPW Method. To incorporate the pressure sensor-based NPW method, pressure sensors are installed upstream and downstream to collect NPW signals to detect leaks. According to the time difference for the detected signals and the propagation velocity in the medium, the exact position of the leak point can be calculated. The principle of the pressure sensor-based NPW method is illustrated in Figure 1.

In Figure 1, the distance between two pressor sensors is $L$, the propagation velocity of the NPW in the pipeline is $v$, the distance between the leak point and the upstream sensor is $X$, the times when the wave is detected by the two sensors are $t_{1}$ and $t_{2}$, and the velocity of natural gas in the pipeline is $u$.

As the pipeline diameter and gas transportation velocity increase, the velocity of the natural gas cannot be ignored compared with that of the NPW. With the velocity of natural gas taken into consideration in our study, the relations between the length and time variables can be developed as follows:

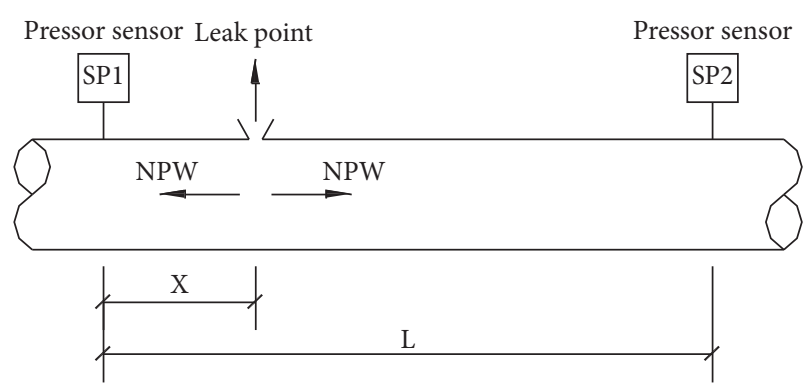

FIgURE 1: Schematic of pressure sensor-based NPW method.

$$
\begin{gathered}
t_{1}=\frac{X}{v-u}, \\
t_{2}=\frac{L-X}{v+u}, \\
\Delta t=t_{1}-t_{1} .
\end{gathered}
$$

The distance between the leak point and the upstream sensor can be obtained from the above three equations:

$$
X=\frac{1}{2 v}\left[L(v-u)+\Delta t\left(v^{2}-u^{2}\right)\right] .
$$

Equation (2) is the leak location formula.

2.2. The law of NPW Propagation and Attenuation. Wave propagation is a form of energy transmission and the NPW is no exception. Energy loss is inevitable in this process and the mathematical model of NPW attenuation [27] is

$$
\left|\Delta P_{x}\right|=\left|\Delta P_{0}\right| e^{-\eta x}
$$

where $\left|\Delta P_{0}\right|$ is the value of the NPW at the leak point, $\left|\Delta P_{x}\right|$ is the value of the NPW at the point that is a distance of $x$ from the leak point, and $\eta$ is the attenuation coefficient. $\eta$ is mainly determined by the internal diameter of the gas pipeline hydraulic friction factor gas compression factor NPW velocity and other factors. It is easy to see from equation (3) that the value of the NPW is inversely proportional to $x$.

As shown in Figure 1, when leakage occurs at the leak point according to the attenuation law of the NPW, the following can be obtained:

$$
\begin{aligned}
& \left|\Delta P_{1}\right|=\left|\Delta P_{0}\right| e^{-\eta X}, \\
& \left|\Delta P_{2}\right|=\left|\Delta P_{0}\right| e^{-\eta(L-X)} .
\end{aligned}
$$

The pressure values measured by SP 1 and SP 2 before and after the occurrence of the leak are recorded. The difference between the two values is the pressure value of the NPW at these two points, namely, $\left|\Delta P_{1}\right|$ and $\left|\Delta P_{2}\right|$. Moreover, $X$ and $L$ are known. Therefore, the pressure value of the NPW at the leak point $\left|\Delta P_{0}\right|$ can be obtained by combining equations (4) and (5).

According to equations (4) and (5), it can be seen that the further away from the leak point, the greater the attenuation 
of the NPW is and thus the smaller the pressure value of the NPW is. In contrast, the closer it is to the leak point, the smaller the attenuation of the NPW will be and thus the greater the pressure value of the NPW will be. The pressure value of the NPW is inversely proportional to the distance from the leak point.

2.3. Minimum Detectable Pressure Value of the Pressure Sensor. Pressure sensors are typically installed at the beginning and end of a section of pipe, as shown in Figure 1. The necessary condition for successful leak detection is that the pressure value of the NPW at these two sensors should be greater than the minimum detectable pressure value of these two sensors. That is, it satisfies the following inequality:

$$
\begin{aligned}
& \left|\Delta P_{1}\right|>\left|\Delta P_{1}\right|_{\text {min }}=\lambda_{1} \sqrt{h_{1}^{2}+\sigma_{1}^{2}}, \\
& \left|\Delta P_{2}\right|>\left|\Delta P_{2}\right|_{\text {min }}=\lambda_{2} \sqrt{h_{2}^{2}+\sigma_{2}^{2}},
\end{aligned}
$$

where $\left|\Delta P_{1}\right|_{\text {min }}$ and $\left|\Delta P_{2}\right|_{\text {min }}$ are the minimum detectable pressure values of SP1 and SP2, $\lambda_{1}$ and $\lambda_{2}$ are the sensitivity coefficients of SP1 and SP2, respectively, and $\sigma_{1}$ and $\sigma_{2}$ are the standard deviation of noise at these two points $h_{1}$ and $h_{2}$ which are the measurement errors of SP1 and SP2. $h_{1}$ and $h_{2}$ can be calculated by the following equations:

$$
\begin{aligned}
& h_{1}=\left(H_{1, \max }-H_{1, \min }\right) \delta_{1}, \\
& h_{2}=\left(H_{2, \max }-H_{2, \min }\right) \delta_{2},
\end{aligned}
$$

where $H_{i, \max }$ and $H_{i, \min }$ represent the upper and lower limits of the sensor range, respectively, and $\delta_{1}$ and $\delta_{2}$ represent the precision of SP1 and SP2. For certain sensors, $h_{1}$ and $h_{2}$ are constant. $\sigma_{1}$ and $\sigma_{2}$ can be calculated from actual data.

It can be seen from the abovementioned analysis that the minimum detectable pressure values of the sensors are determined by the precision of the sensors, the upper and lower limits of the sensor, and other parameters. Once the sensors are determined, the minimum detectable pressure values are determined.

\subsection{Disadvantages of Pressure Sensor-Based NPW Method.} According to the abovementioned study and the principle of fluid mechanics, the key to leak detection is whether the pressure values of the NPW at the sensors are greater than the minimum detectable pressure values of the sensors. The pressure values of the NPW at the sensors depend on the pipeline operating pressure, the leakage rate, and the distance between the leak point and the sensors. These pressure values are inversely proportional to the distance between the leak point and the sensors and are directly proportional to the operating pressure and the leakage rate. Among them, the operating pressure and leakage rate are uncontrollable which makes the distance between the leak point and the sensors a key factor affecting the pressure value of the NPW.

Pressure sensors are used to detect the NPW in this method. Since the installation of pressure sensors requires altering, the pipeline structure pressure sensors are usually installed at the head and end of a pipeline or at compressor stations before the pipeline is placed into operation. Once the pipe is running, it is difficult to install pressure sensors. As a result, pressure sensors are typically placed far apart. When a leak occurs, the distance between the leak point and the pressure sensors is generally far. According to the analysis in Section 2.2, the pressure values of an NPW exhibit large attenuations at this time. If the pipeline operating pressure or leakage rate is low at this time, the pressure values of the NPW at the sensors are easily less than the minimum detectable pressure values of the pressure sensors and the NPW will be difficult to detect. This is the reason why the pressure sensor-based NPW method is basically ineffective for small leaks and has a high failure rate.

\section{FBG Strain Sensor-Based NPW Method}

Through the abovementioned study, it can be seen that to overcome the shortcomings of the pressure sensor-based NPW method, the pressure value of the NPW at the sensors must be increased. The most direct way to achieve this is to reduce the distance between the leak point and the sensors to reduce the attenuation of the NPW and achieve the purpose of increasing the pressure value of the NPW at the sensors. In this paper, a method based on an FBG strain sensor is proposed to detect the NPW.

\subsection{Using an FBG Strain Sensor to Detect NPW in the} Pipeline. An FBG sensor is a kind of fiber optic sensor in which ordinary light will pass through a grating without being affected by it. Only certain wavelengths of light will be reflected at the grating. The spacing of the grating will change due to changes in external stress or temperature which will also change the wavelength of the light reflected by the grating. Therefore, the strain and temperature changes in the external environment where the grating is located can be detected by monitoring the wavelength change in the reflected light.

Figure 2 shows a schematic diagram of a clamping FBG strain sensor. It can be seen in the figure that this sensor mainly consists of three parts: an FBG, a clamping part, and a fixed end. The FBG is glued and fixed on the clamping part. Assume the distance between the two fixed ends is $I$ and the distance between the two clamping parts is $I_{f}$. The external strain is $\varepsilon$ and the central wavelength of the sensor changes to $\Delta \lambda_{\mathrm{FBG}}$.

According to the material mechanics and optical fiber sensing principle, the relationship between the central wavelength change of the sensors and the external strain can be written as follows:

$$
\varepsilon=\frac{I_{f} \Delta \lambda_{\mathrm{FBG}}}{1.2 I} .
$$

Equation (8) shows that the external strain is directly proportional to the central wavelength change of the sensor, so the external strain can be detected by measuring the central wavelength change of the sensor. 


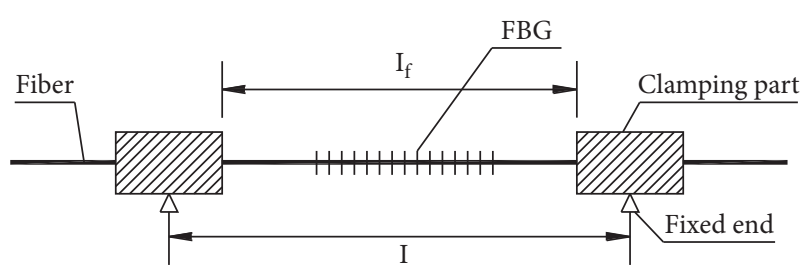

FIGURE 2: Sketch map of clamping FBG strain sensor.

The FBG strain sensors developed in this study are wrapped around the wall of the pipeline, as shown in Figure 3. A change in pressure within the pipeline leads to its expansion or contraction with the annular (circumferential) strain of the pipeline changing accordingly. The FBG strain sensors detect pressure changes within the pipe by sensing the annular strain. The annular strain within a pipeline system can be expressed as

$$
\varepsilon=\frac{\sigma_{y}-v \sigma_{z}}{E}
$$

where $\varepsilon_{y}$ is the pipeline annular strain, $v$ is the pipeline Poisson's ratio, $\sigma_{y}$ is the pipeline annular stress, $\sigma_{z}$ is the pipeline axial stress, and $E$ is the pipeline elasticity modulus.

Using equation (9), a relationship can be derived to relate the annular strain with the pipeline pressure and pipe wall thickness. First, it is assumed that the pipeline is infinitely long, so the axial stress can be neglected, that is, $\sigma_{z}=0$. Meanwhile, as $\sigma_{y}=\mathrm{PD}_{w} / 2 h$, the values for $\sigma_{y}$ and $\sigma_{z}$ can be substituted into equation (9) which gives

$$
\varepsilon=\frac{\mathrm{PD}_{w}}{2 \mathrm{hE}}
$$

where $P$ is the pressure in the pipeline, $D_{w}$ is the external diameter of the pipeline, and $h$ is the pipeline wall thickness.

As seen from equation (10), the annular strain of the pipeline is also directly proportional to the pressure in the pipeline, so the pressure change in the pipe can be monitored by monitoring the annular strain of the pipeline. At this time, the distance between the two fixed fulcrums becomes the perimeter of the pipeline, that is, $I=\pi D_{w}$. Therefore, equation (8) can be expressed as

$$
\varepsilon=\frac{I_{f} \Delta \lambda_{\mathrm{FBG}}}{1.2 I}=\frac{I_{f} \Delta \lambda_{\mathrm{FBG}}}{1.2 \pi D_{w}} .
$$

Equation (11) is combined with equation (10) to obtain the relationship between the central wavelength change of the sensors and the pressure inside the pipeline as follows:

$$
\Delta \lambda_{\mathrm{FBG}}=\frac{0.6 \pi D_{w}^{2}}{\mathrm{hEI}_{f}} P .
$$

Equation (12) shows that the central wavelength change of the sensor is directly proportional to the pressure in the pipeline. Therefore, the pressure in the pipeline can be detected by monitoring the central wavelength of the sensor. This is the operating principle of the FBG strain sensor for detecting pressure changes in the pipeline.
Temperature variations can also affect the annular strain; thus, for actual projects, FBG temperature sensors are needed to compensate for the effect of diurnal or seasonal temperature variations on the annular strain.

\subsection{FBG Strain Sensor-Based NPW Method. According to} the operating principle of the FBG strain sensor, detecting the NPW in pipelines above a leak detection method based on the FBG strain sensor is proposed, as shown in Figure 4. In this method, the FBG strain sensors are set at intervals along the pipeline, so that when a leak occurs in some parts of the pipeline, the leak point is very close to the sensors on both sides. Therefore, the attenuation of the NPW is very small and the pressure value of the NPW at the sensors is relatively large which overcomes the high failure rate of the NPW method based on a pressure sensor. At the same time, since the location of each sensor in this method is known, the calculation method of the leak point location is the same as that of the pressure sensor-based method.

The important reason why FBG strain sensors can be set at shorter intervals is that the installation of these sensors is simple requiring only the partial removal of the anticorrosion layer and adhering the sensor to the outer wall of the pipeline without destroying the pipeline structure. In addition, it is easy to add FBG strain sensors to an existing pipeline, thus realizing nondestructive installation. The cost is also lower. In addition, FBG strain sensors have the advantages of being insensitive to interference signals and exhibiting small attenuations along their traversed length.

\subsection{Experiment}

3.3.1. Experimental Platform. The experimental platform for testing the FBG strain sensor-based NPW method is outlined in this paper. The schematic and photo for this platform are shown in Figure 5. Two air tanks and a section of pipeline were used to simulate a realistic gas transfer. The pipeline in this experiment was made of steel with a diameter of $273 \mathrm{~mm}$ as frequently used in practice. The pipeline length was $11 \mathrm{~m}$ due to lab space limitations. A leak point was simulated by manually opening a valve at different locations, a rotameter was located at the leak point to measure the leak rate, and two FBG strain sensors (SF1 and SF2) and two pressure sensors (SP1 and SP2) were installed.

For safety considerations, the maximum design pressure of this platform was $1.0 \mathrm{MPa}$ and air was used in this experiment instead of natural gas. In this leak detection experiment, we were concerned with physical changes to the pipeline and the chemical properties of natural gas were not involved, so this replacement was appropriate. In addition, due to the short leak time and the constant room temperature, there was no need to consider the effects of temperature variations on the FBG strain sensor or leak detection; thus, there were no temperature sensors installed on this platform. 


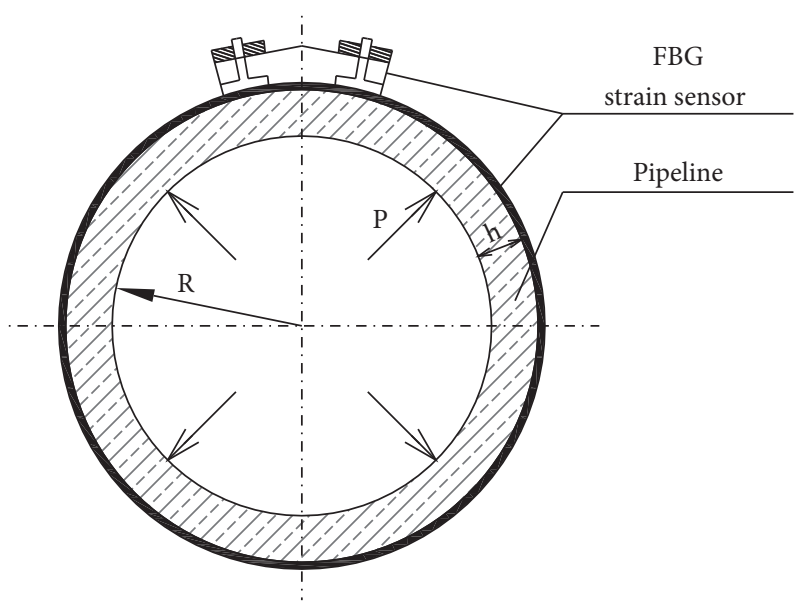

(a)

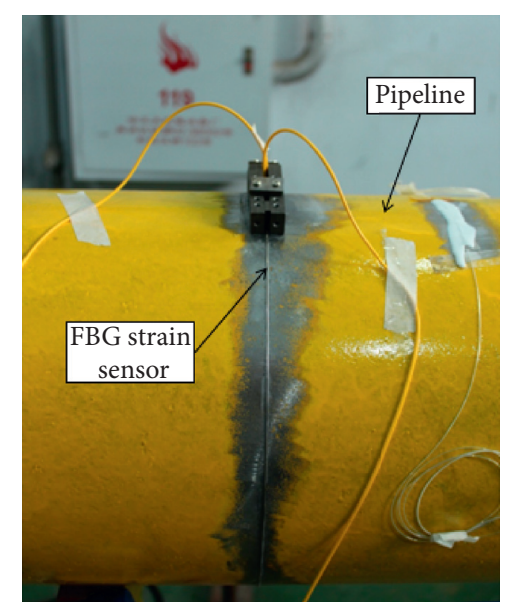

(b)

FIgURE 3: (a) Schematic of the FBG strain sensor. (b) Photo of a sensor mounted on a pipeline.

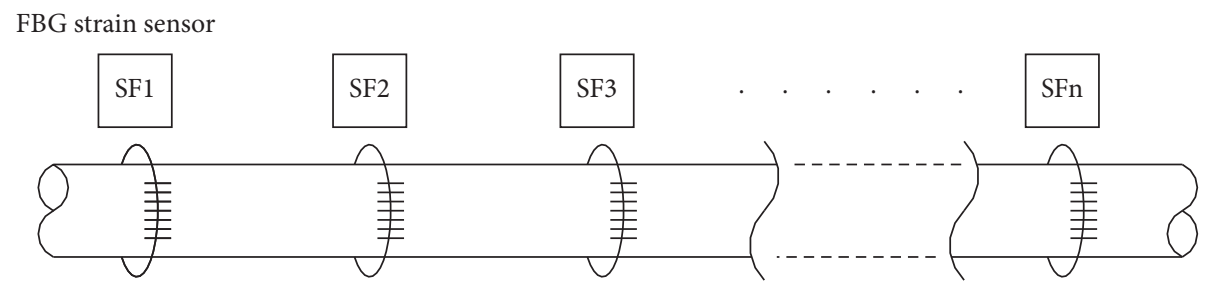

FIGURE 4: Schematic of FBG strain sensor-based NPW method.

3.3.2. Calibration of the FBG Strain Sensors. The FBG strain sensors (SF1 SF2) were calibrated to help determine the relationship between pipeline pressure and wavelength change. The pipeline was pressurized by a compressor from $0 \mathrm{kPa}$ to $800 \mathrm{kPa}$ in approximately $50 \mathrm{kPa}$ increments per step. At each pressure level, the wavelength changes at the fiber receptor were measured. Two calibration test results are plotted in Figure 6, showing the relationship between wavelength change and pressure. It is obvious that the linearity of the pressure-to-wavelength change is excellent and the coefficient of linear association is more than 0.999 which agrees well with the theoretical analysis mentioned above. In accordance with the equation represented in Figure 6, the sensitivity coefficients of these two sensors can also be obtained which are $0.088 \mathrm{pm} / \mathrm{kPa}$ and $0.091 \mathrm{pm} / \mathrm{kPa}$, respectively.

3.4. Experimental Test and Comparison. The FBG sensors (SF1 and SF2) were tested by the experimental platform and the results were compared with those of the pressure sensors (SP1 and SP2). Briefly, the experimental process followed these four steps: (1) air is compressed into air tank AT1 by air compressor AC. AT1 plays the role of stabilizing the pressure in the pipeline. (2) When the pressure is stable, solenoid valve SV1 is opened to allow the flow of air into the pipeline from AT1 to AT2. (3) Ball valve BV4 at the leak point is opened to simulate a leak in the pipeline. In the meantime, all the sensors gather data. (4) After a period of time, ball valve BV4 is closed and the experiment ends.

Figure 7 shows the pressure signal collected by FBG strain sensors SF1 and SF2. As seen in the figure, the pressure in the pipeline is stable before the leak occurs. When ball valve BV4 is opened and the pipeline leaks, the measured pressure signal in the pipeline drops suddenly. This is because the NPW generated by the leak propagates to both sides of the leak point, resulting in pressure drops at the positions of SF1 and SF2 which further causes changes in the annular strain of the outer wall of the pipeline. Strain changes are detected by SF1 and SF2 and thus pressure changes within the pipe are detected. This is consistent with the NPW theory. Figure 7 also shows that after a sudden drop in the pressure waveform, a small rise in the pressure tends to level off. This is because the leak only lasted for a short period of time in the experiment. When ball valve BV4 is closed to stop the leak, the water hammer phenomenon will be formed in the pipeline, causing a rise in the pressure in the pipeline and forming a small shock which will gradually stabilize. However, because of the loss of gas, the restored steady pressure was lower than the initial pressure. This result fully shows that it is feasible to use FBG strain sensors to collect pressure signals in the pipeline and detect gas pipeline leaks.

Figure 8 shows the pressure signal collected by pressure sensors SP1 and SP2. The pressure change trend is consistent with that collected by pressure sensors SF1 and SF2. 


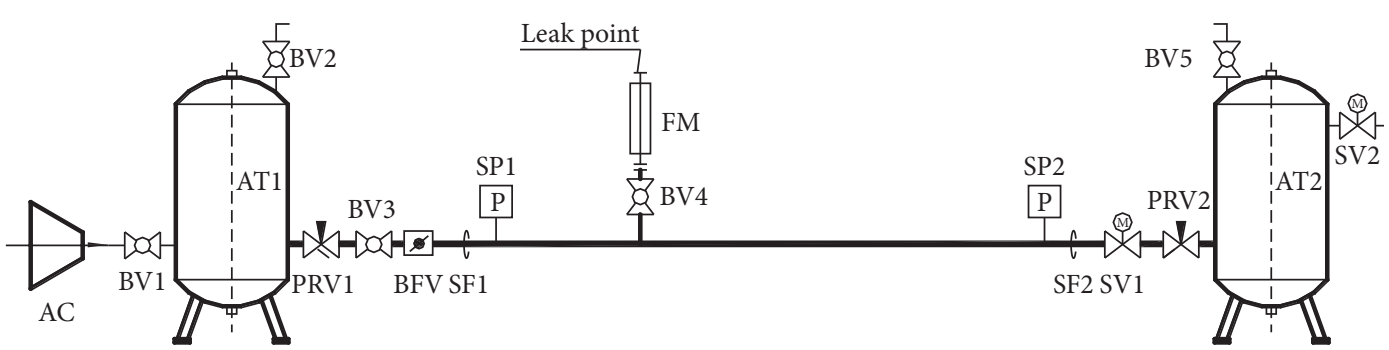

(a)

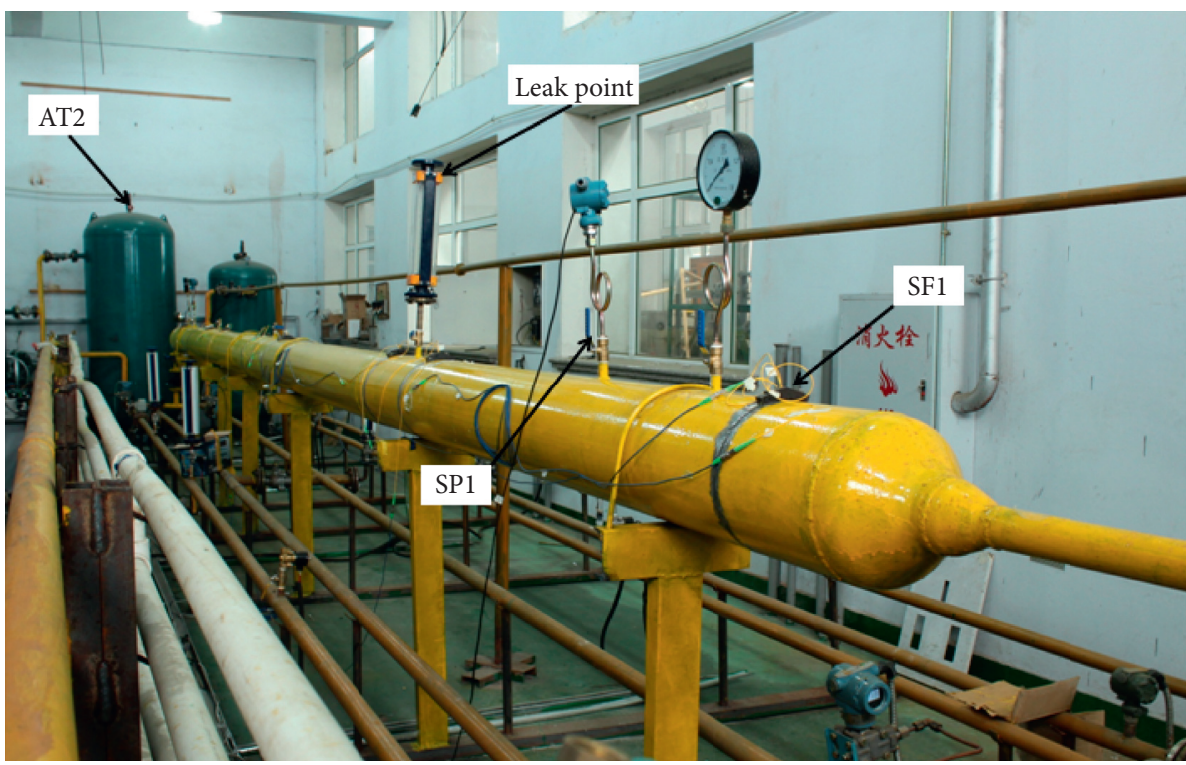

(b)

FIGURE 5: (a) System diagram of the experiment platform, AC, air compressor; AT1 AT2, air tank; SP1 SP2, pressure sensor; SF1 SF2, FBG strain sensor; BFV, butterfly valve; BV1 BV5, ball valve; SV1 SV2, solenoid valve; FM, flowmeter; PRV1 PRV2, pressure-regulating valve. (b) Photo of experiment platform.

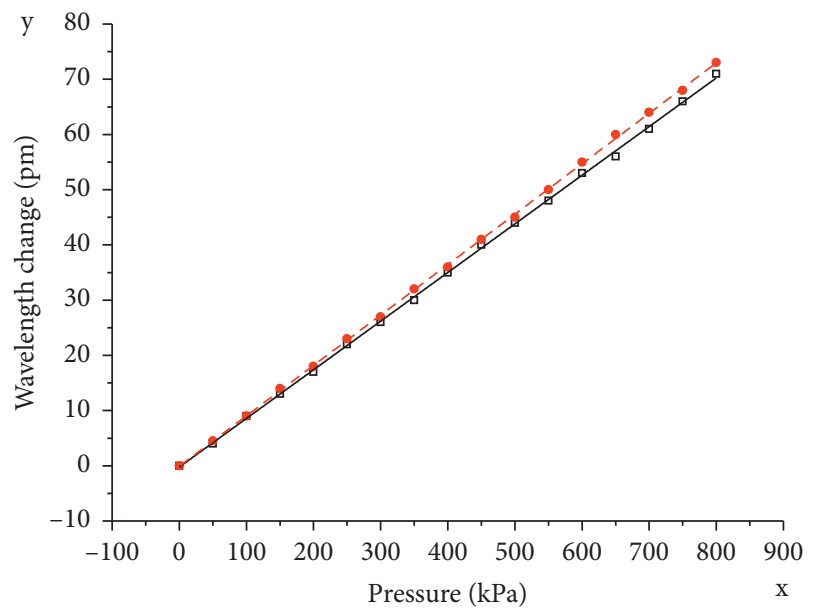

- FBG strain sensor SF1

_ Linear fit of SF1 $\mathrm{y}=0.088 \mathrm{x}-0.012$ $R^{2}=0.999423$

- FBG strain sensor SF2

- - - Linear fit of SF2 $\mathrm{y}=0.091 \mathrm{x}-0.019$, $R^{2}=0.999166$

Figure 6: Calibration of FBG strain sensors (SF1 SF2).
Figures 7 and 8 can be used to compare the NPW detection effect of the FBG strain sensor and the pressure sensor. First, as can be clearly seen in the two figures, the noise of the signal captured by pressure sensors is significantly higher than the signal captured by the FBG strain sensors. Second, the noise of the signal captured by the pressure sensor SP2 is significantly higher than SP1. This is because the distance between the leak point and SP1 is shorter than the distance between the leak point and SP2. However, additional noise is not significantly present in FBG strain sensor SF2 compared with SF1 even though SF2 is farther from the leak than SF1. Therefore, the noise of the pressure sensors increases with greater distance from the leak point but the FBG strain sensors are less affected by this factor.

These results indicate that (1) FBG strain sensors filter out interference much more than pressure sensors and (2) the attenuation of FBG strain sensors is much smaller than that of pressure sensors. In addition to the above two advantages, FBG strain sensors also have the advantages of simple and nondestructive installation. Therefore, the sensors can be arranged at shorter intervals along the pipeline. This addresses the problems of large signal noise and the ineffective detection of small leaks inherent in the pressure sensor-based NPW method. 

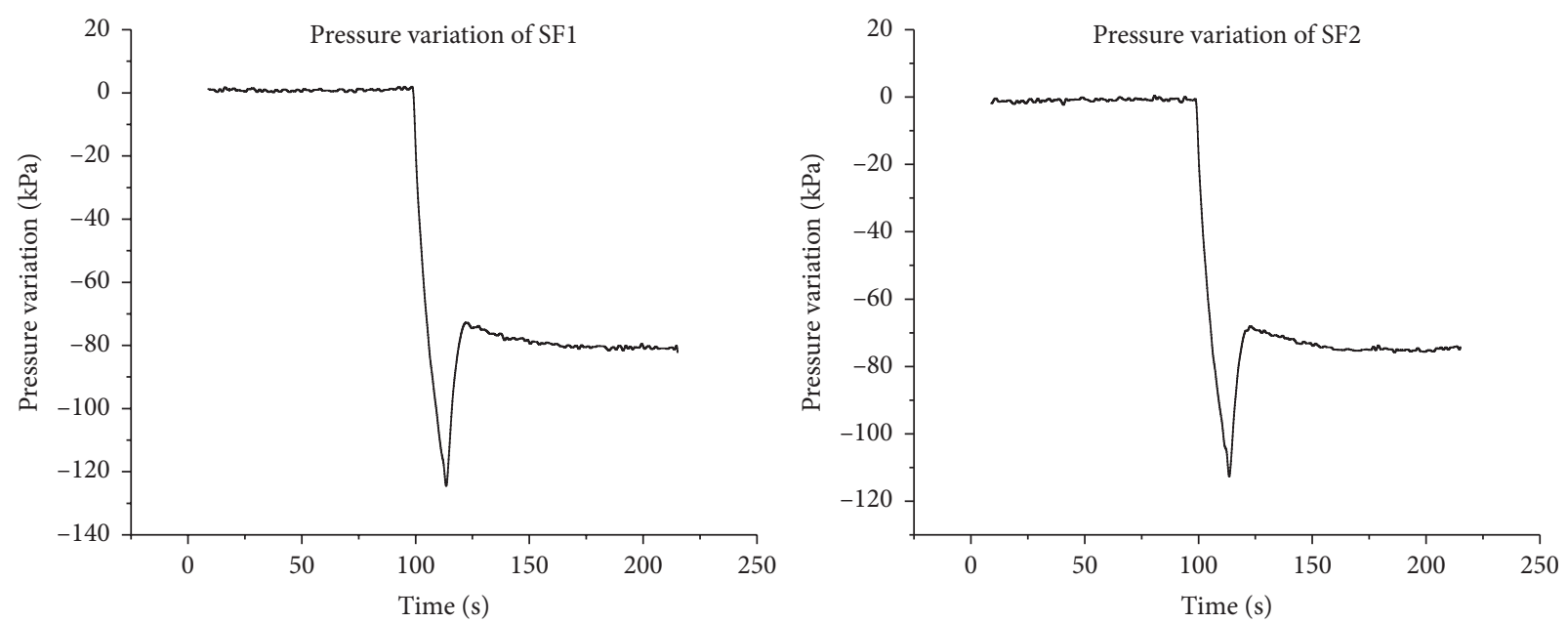

FIgURE 7: Signal captured by SF1 and SF2.
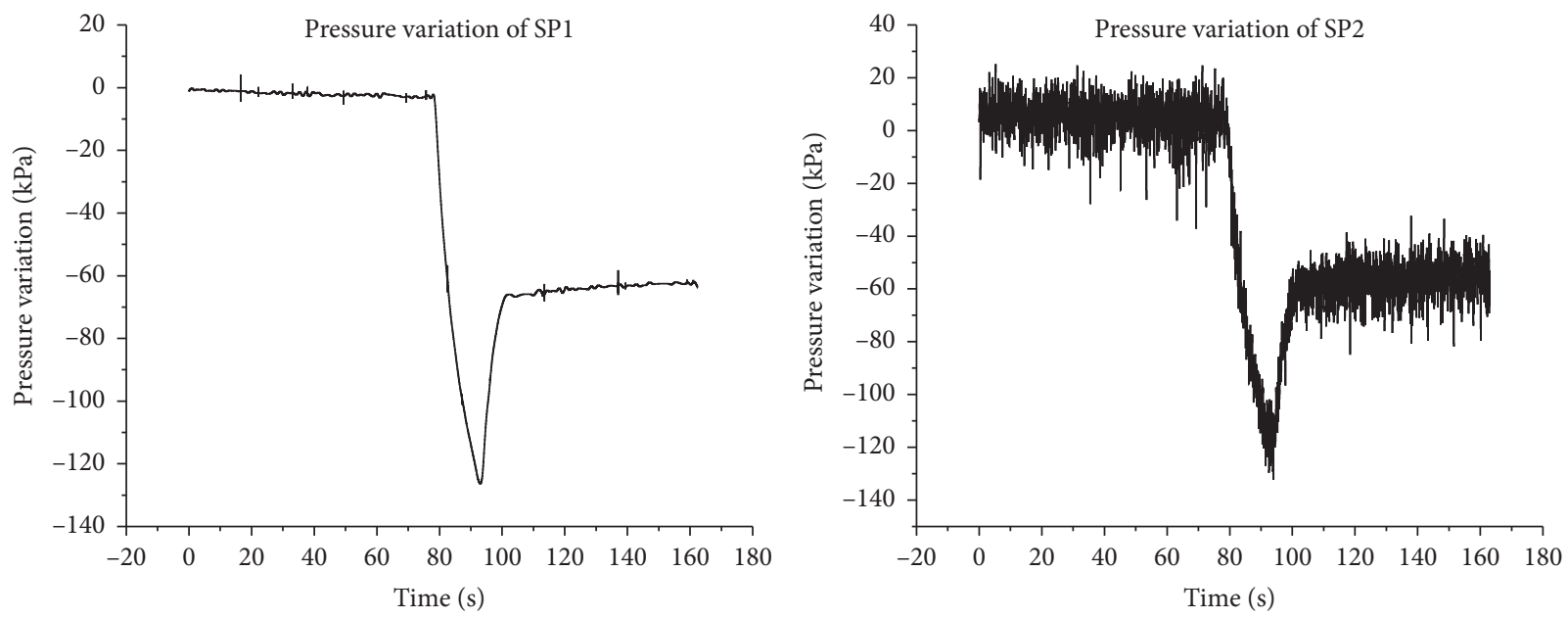

FIgURE 8: Signal captured by SP1 and SP2.

These results demonstrate that the FBG strain sensorbased NPW method is more suitable for natural gas pipeline leak detection.

\section{Conclusion}

In this paper, the propagation and attenuation of NPWs in natural gas pipelines are introduced, the principle of detecting and locating leaks by the pressure sensor-based NPW method is studied, and the disadvantages of this method are noted. Then, in view of the shortcomings of the pressure sensor-based NPW method, a method of detecting the NPW in a pipeline by using an FBG strain sensor to monitor the annular strain of the pipeline is proposed and a method based on the FBG strain sensor to detect and locate pipeline leaks is proposed. Finally, through experimental tests and comparisons, it is concluded that using an FBG strain sensor to detect an NPW has the advantages of nondestructive installation of high sensitivity and low interference. Therefore, the FBG strain sensor-based NPW method is easier to install and sensitive to small leaks and low noise, so it is more suitable for leak detection in natural gas pipelines.

As mentioned in Section 3.3.1, the laboratory temperature is relatively constant, thus the effect of temperature variations on the FBG strain sensor and leak detection is not considered in this paper. However, in practical engineering, the natural gas pipelines often span a wide range and have large temperature variation. The influence of temperature variation on leak detection should be further studied.

\section{Data Availability}

The data used to support the findings are available upon request. 


\section{Conflicts of Interest}

The author declares no conflicts of interest.

\section{Acknowledgments}

This research was funded by the Doctoral Research Project of Harbin University of Commerce (no. 2016BS19) and Project of Harbin Science and Technology (no. 2016RAQXJ023). This work was also partially supported by the Fundamental Research Funds for the National Natural Science Foundation of China (no. 51978202), the Natural Science Fund in Heilongjiang Province (no. LH2020E028), and Science Foundation of Harbin University of Commerce (no. 18XN068).

\section{References}

[1] H. R. Zhang, Y. T. Liang, W. Zhang, N. Xu, Z. L. Guo, and G. M. Wu, "Improved PSO-based method for leak detection and localization in liquid pipelines," IEEE Transactions on Industrial Informatics, vol. 14, pp. 3143-3154, 2018.

[2] H. R. Zhang, Y. T. Liang, Q. Liao, S. Yun, and X. H. Yang, “A self-learning approach for optimal detailed scheduling of multi-product pipeline," Journal of Computational and Applied Mathematics, vol. 327, pp. 41-63, 2018.

[3] Q. Liao, H. R. Zhang, N. Xu, Y. T. Liang, and J. N. Wang, "A MILP model based on flowrate database for detailed scheduling of a multi-product pipeline with multiple pump stations," Computers \& Chemical. Engineering.vol. 11, pp. 763-781, 2018.

[4] Q. Liao, P. M. Castro, Y. T. Liang, and H. R. Zhang, "New batch-centric model for detailed scheduling and inventory management of mesh pipeline networks," Computers \& Chemical. Engineering, vol. 130, no. 2, Article ID 106568, 2019.

[5] Z. B. Li, H. X. Feng, Y. T. Liang, N. Xu, S. M. Nie, and H. R. Zhang, "A leakage risk assessment method for hazardous liquid pipeline based on Markov chain Monte Carlo," International Journal of Critical Infrastructure Protection, vol. 27, 2019.

[6] B. Wang, Y. Liang, J. Zheng, T. Lei, M. Yuan, and H. Zhang, "A methodology to restructure a pipeline system for an oilfield in the mid to late stages of development," Computers \& Chemical Engineering, vol. 115, pp. 133-140, 2018.

[7] I. R. Ellul, "Advances in pipeline leak detection techniques," Pipes and Pipelines International, vol. 34, pp. 7-12, 1989.

[8] R. A. Silva, C. M. Buiatti, S. L. Cruz, and J. A. F. R. Pereira, "Pressure wave behavior and leak detection in pipelines," Computers and Chemical Engineering, vol. 20, pp. 491-496, 1996.

[9] B. Vogel, C. Cassens, A. Graupner, and A. Trostel, "Leakage detection systems by using distributed fiber optical temperature measurement," SPIE, vol. 4328, pp. 23-34, 2001.

[10] E. A. Mendoza, R. A. Lieberman, J. D. Prohaska, and D. P. Robinson, "Distributed fiber optic chemical sensors for detection of corrosion in pipelines and structural components," SPIE, vol. 3398, pp. 136-143, 1998.

[11] O. S. Wolfbeis, "Fiber-optic chemical sensors and biosensors," Analytical Chemistry, vol. 78, no. 12, pp. 3859-3874, 2006.

[12] C. McDonagh, C. S. Burke, and B. D. MacCraith, "Optical chemical sensors," Chemical Reviews, vol. 108, no. 2, pp. 400-422, 2008.
[13] D. S. Mekeehan, R. W. Griffiths, and J. E. Halkyard, "Marine application for a continuous fiber optic strain monitoring system," in Proceedings of the the 18th Annual OTC, pp. 342-345, Houston, TX, USA, May 1986.

[14] S. Yashiro, T. Okabe, and N. Takeda, "Damage identification in a holed CFRP laminate using a chirped fiber Bragg grating sensor," Composites Science and Technology, vol. 67, no. 2, pp. 286-295, 2007.

[15] H. Tsuda, J.-R. Lee, and Y. Guan, "Fatigue crack propagation monitoring of stainless steel using fiber Bragg grating ultrasound sensors," Smart Materials and Structures, vol. 15, no. 5, pp. 1429-1437, 2006.

[16] D. C. Betz, G. Thursby, B. Culshaw, and W. J. Staszewski, "Advanced layout of a fiber Bragg grating strain gauge rosette," Journal of Lightwave Technology, vol. 24, no. 2, pp. 1019-1026, 2006.

[17] E. Liu, L. Lv, Y. Yi, and P. Xie, "Research on the steady operation optimization model of natural gas pipeline considering the combined operation of air coolers and compressors," IEEE Access, vol. 7, Article ID 83251, 2019.

[18] E. Liu, X. Ma, and M. Zhou, "Analysis of discharge process of oil pipeline with complex topography," Energy Reports, vol. 5, pp. 678-687, 2019.

[19] J. Zheng, Y. Dai, Y. Liang, Q. Liao, and H. Zhang, "An online real-time estimation tool of leakage parameters for hazardous liquid pipelines," International Journal of Critical Infrastructure Protection, vol. 31, Article ID 100389, 2020.

[20] H. Lu, T. Iseley, S. Behbahani, and L. Fu, "Leakage detection techniques for oil and gas pipelines: state-of-the-art," Tunnelling and Underground Space Technology, vol. 98, Article ID 103249, 2020.

[21] H.-J. Park and M. Song, "Linear FBG temperature sensor interrogation with Fabry-Perot ITU multi-wavelength reference," Sensors, vol. 8, no. 10, pp. 6769-6776, 2008.

[22] N. Takeda, Y. Okabe, J. Kuwahara, S. Kojima, and T. Ogisu, "Development of smart composite structures with small-diameter fiber Bragg grating sensors for damage detection: quantitative evaluation of delamination length in CFRP laminates using Lamb wave sensing," Composites Science and Technology, vol. 65, no. 15-16, pp. 2575-2587, 2005.

[23] A. Dandridge and C. Kirkendall, "Passive fiber optic sensor networks," in Handbook of Optical Fiber Sensing Technology, J. M. López-Higuera, Ed., pp. 433-448, Wiley, New York, NY, USA, 2002.

[24] H. Tsuda, "Ultrasound and damage detection in CFRP using fiber Bragg grating sensors," Composites Science and Technology, vol. 66, no. 5, pp. 676-683, 2006.

[25] Q. M. Hou, W. L. Jiao, L. Ren, H. Z. Cao, and G. B. Song, "Experimental study of leakage detection of natural gas pipeline using FBG based strain sensor and least square support vector machine," Journal of Loss Prevention in the Process Industries, vol. 8, pp. 56-69, 2014.

[26] R. M. López, V. V. Spirin, M. G. Shlyagin et al., "Coherent optical frequency domain reflectometry for interrogation of bend-based fiber optic hydrocarbon sensors," Optical Fiber Technology, vol. 10, no. 1, pp. 79-90, 2004.

[27] A. B. Sergey, F. L. Oleg, and Energoavtomatika Ltd, "Waves Attenuation and the Pressure Surge Method Performance," in Proceedings of the PSIG Annual Meeting, \Alberta, Canada, October 2007. 